\title{
Le théâtre de Mandeure. Restitution, fonction, datation
}

Séverine Blin et Jean-Yves Marc

\section{(2) OpenEdition}

1 Journals

Édition électronique

URL : http://journals.openedition.org/edl/82

DOI : $10.4000 /$ edl. 82

ISSN : 2296-5084

Éditeur

Université de Lausanne

Édition imprimée

Date de publication : 15 mai 2011

Pagination : 47-72

ISBN : 978-2-940331-25-3

ISSN : 0014-2026

\section{Référence électronique}

Séverine Blin et Jean-Yves Marc, "Le théâtre de Mandeure. Restitution, fonction, datation », Études de lettres [En ligne], 1-2 | 2011, mis en ligne le 15 mai 2014, consulté le 19 décembre 2020. URL : http:// journals.openedition.org/edl/82 ; DOI : https://doi.org/10.4000/edl.82 


\section{LE THÉÂTRE DE MANDEURE RESTITUTION, FONCTION, DATATION}

La reprise des recherches au théâtre de Mandeure a permis d'en modifier de manière substantielle le plan et l'histoire. Sous le gigantesque théâtre actuellement visible, dont le plan et l'apparat monumental apparaissent de plus en plus comme très "canoniques", on a pu mettre au jour les vestiges d'au moins deux édifices de spectacle, dont le plus ancien aurait pu être équipé d'un dispositif scénique très différent d'une orchestra. Mais nous sommes encore loin de restituer, comme à Augst, trois phases claires et bien datées. Nous devrons poursuivre nos recherches en tenant également compte du renouvellement complet de nos connaissances sur le sanctuaire dans lequel s'insère dès l'origine l'édifice de spectacle.

\section{Introduction}

Les trois opérations annoncées dans notre titre, restitution, fonction et datation, sont indissociables les unes des autres dans l'étude d'un édifice de spectacle. On sait combien les transformations et les remaniements des plans et des élévations des édifices de spectacle des Gaules sont liés à l'évolution du goût des spectacles mais aussi aux exigences politiques et cultuelles. Comment concevoir en effet la restitution d'un dispositif scénique sans le préalable nécessaire que constitue la réflexion sur sa fonction? Comment restituer tel ou tel état de construction, si les questions de chronologie relative des différentes structures visibles n'ont pas été résolues? Ces questions de méthode qui devraient aller de soi, ne sont malheureusement pas toujours prises en compte avec toute la rigueur nécessaire. La difficulté principale dans l'appréhension 
de ces monuments réside tout d'abord dans leur ampleur. Ils figurent parmi les plus imposants de la panoplie monumentale des agglomérations antiques. Elle réside ensuite dans la multiplication d'états de construction très nombreux ou de remaniements disparates, hétérogènes et d'ampleurs inégales, qui sont trop souvent saisis partiellement, faute d'exploration exhaustive et systématique. L'ouverture de sondages limités est certainement la stratégie la plus usitée mais aussi la moins adaptée aux édifices de spectacle.

De ce point de vue, le cas du théâtre de Mandeure est malheureusement exemplaire. Considéré depuis Arcisse de Caumont comme le modèle type du "théâtre gallo-romain", il a souffert d'une exploration très incomplète, d'une confusion des différents états de construction et de certitudes erronées sur la fonction, comme sur le plan. Il s'avère désormais que ce qui passait hier pour constituer les caractéristiques mêmes du «théâtre gallo-romain " - absence ou modestie de la décoration architecturale des façades, atrophie ou absence de dispositif scénique, absence de mur de scène - s'explique davantage par l'inachèvement de l'exploration archéologique et l'absence d'une autopsie exhaustive et systématique des vestiges. Dans le cas du théâtre de Mandeure, l'édifice présente au moins trois grandes phases de construction, chacune correspondant à un projet d'architecture significativement différent de l'autre. Mais rien ne dit pour le moment que chacun de ces trois édifices de spectacle ait correspondu à cette catégorie "topique" ou "ethnique" du "théâtre galloromain». Pas plus à Mandeure qu'ailleurs, le classement d'un édifice de spectacle dans cette catégorie monumentale artificielle ne résout les problèmes liés à la restitution ou à la fonction de l'édifice. Ajoutons, enfin, que le type du théâtre gallo-romain repose tout à la fois sur des préjugés culturels, sur des caractéristiques architecturales qui ont été remises en cause ces trente dernières années et, enfin, sur une documentation très inégalement constituée. Qui peut penser aujourd'hui que les provinces des Gaules et des Germanies n'étaient pas suffisamment romanisées pour voir se développer une architecture théâtrale semblable à celle que l'on observe dans les autres provinces de l'Empire? Qui peut encore aujourd'hui soutenir l'opposition stricte entre un idéal type architectural, réservé à l'Urbs et aux provinces les plus romanisées, et ses avatars hybrides ou incomplets construits dans les provinces "périphériques"? Lorsque l'on se penche sur les plans connus et publiés des théâtres des provinces occidentales, on est frappé par la grande variété typologique 
des édifices de spectacle du monde romain, variété typologique qui est loin de se laisser réduire dans des catégories topiques ou ethniques. Enfin, les progrès des recherches sur les théâtres dans les Gaules et les Germanies ces trente dernières années (à Argentomagus, Augst, Avenches ou encore Dalheim), montrent que la documentation sur laquelle raisonnait Albert Grenier, par exemple, n'autorise guère de certitudes, pas de celles en tout cas qui fondent des typologies sérieuses ${ }^{1}$. En ce qui concerne Mandeure, il y a de moins en moins de points communs entre le plan du théâtre publié par Frédéric Morel-Macler au milieu du $\mathrm{XIX}^{\mathrm{e}}$ siècle et celui que nous proposons aujourd'hui.

\section{Le complexe monumental du théâtre et du sanctuaire}

Grâce aux prospections géophysiques et aux fouilles du Programme Collectif de Recherches "Approche pluridisciplinaire d'une agglomération antique, Epomanduodurum (Mandeure-Mathay, Doubs) Archéologie territoire et environnement» menées depuis 2001 sur le site, le renouvellement de nos connaissances, tant sur l'urbanisme de la ville que sur le quartier monumental où se trouve le théâtre, a été spectaculaire. L'agglomération de Mandeure s'étend à l'intérieur de la boucle du Doubs sur la superficie remarquable de 180 ha urbanisés (fig. 1). A l'intérieur de cette vaste trame viaire, le complexe monumental se résumait, depuis le XIX siècle, à un face à face visuel entre le théâtre et le sanctuaire et semblait se situer plutôt en périphérie de l'agglomération antique. Sur ce point, le plan levé en 1893 par la Société d'Emulation de Montbéliard n'avait guère évolué. Depuis 2003, ce secteur s'est révélé être une aire sacrée cernée d'un mur d'enceinte, qui se développe désormais sur plus de 15 ha (fig. 2). Le nombre de vestiges architecturaux reconnus a été également considérablement augmenté. Cet espace cultuel s'organise en effet autour d'un théâtre et de plusieurs temples ou groupement de temples, accompagnés d'autels, de chapelles ou d'annexes cultuelles. Le sanctuaire de Mandeure rejoint par voie de conséquence

I. Plusieurs auteurs ont déjà commenté cette typologie et ses incohérences, voir par exemple F. Dumasy, "Les édifices théâtraux de type gallo-romain, essai de définition"; E. Frézouls, "Aspects de l'histoire architecturale du théâtre romain" et M. Matter, «Particularités architecturales des édifices de spectacles en Gaule lyonnaise». 
Fig. 1 - Plan de l'agglomération antique de Mandeure.

ce type de zone monumentale qualifiée par Pierre Gros «d'enceinte à édifices multiples", connu dans toute la Gaule et dont quelques exemples fameux sont situés non loin de là, en territoire helvète (Avenches) et trévire (Altbachtal) ${ }^{2}$. Comme on s'en doute, ce contexte monumental éclaire d'un jour nouveau la fonction du théâtre.

\section{Histoire des recherches ${ }^{3}$}

Les premières recherches archéologiques sur le théâtre de Mandeure furent réalisées sous le contrôle du sous-préfet de Montbéliard, le comte de Montrond, et sous la direction de Frédéric Morel-Macler, architecte

2. S. Blin, "Architecture et société à Mandeure au Haut-Empire».

3. Une publication de l'histoire de la découverte du site et des fouilles est en préparation sous la direction de Jean-Yves Marc et Pierre Mougin. 
Fig. 2 - Plan du sanctuaire. 


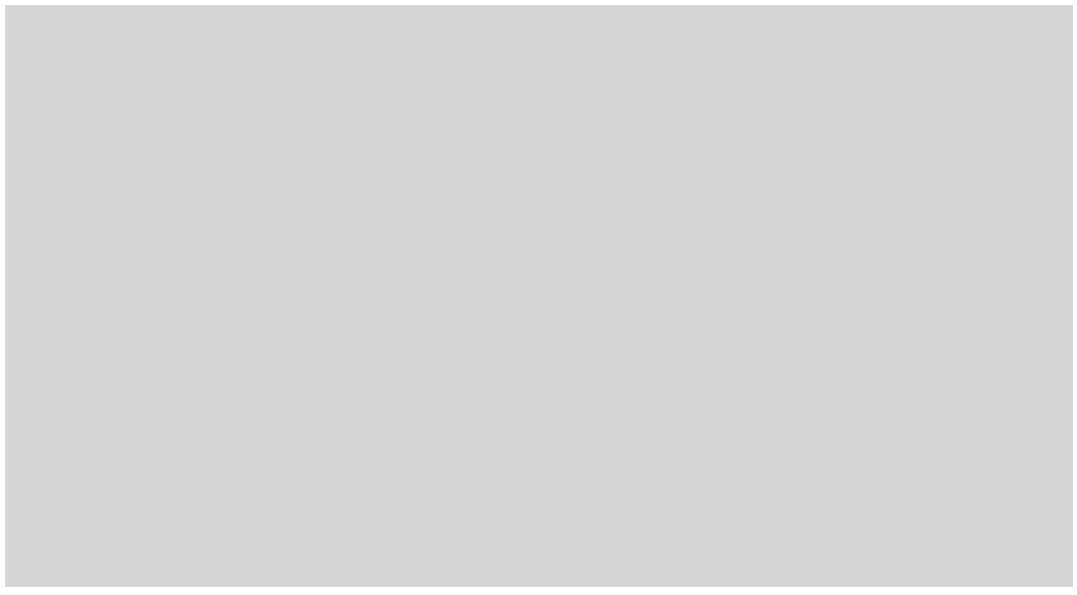

Fig. 3 - Plan du théâtre levé par Frédéric Morel-Macler en 1820.

de la ville de Montbéliard, entre 1817 et 1821 . L'édifice fut très rapidement connu, suffisamment en tout cas pour attirer les voyageurs et faire l'objet des premières gravures publiées (voir par exemple le guide de Charles Nodier en 1825 ou à peine plus tard, en 1828, celui de Philippe de Golbery). Au début du mois de juin 1836, Prosper Mérimée, alors inspecteur des Monuments historiques, visite Mandeure en compagnie de Frédéric Morel-Macler. Il est très impressionné par la qualité de l'édifice, même s'il le range dans les monuments de la décadence romaine. En août 1843, il revient en inspection sur le site. C'est probablement alors que le théâtre fait l'objet d'un classement aux Monuments historiques, ce qui fait de lui l'un des premiers édifices protégés de la région. Ce classement entraîne également de nombreux travaux de restauration nécessaires à la conservation de l'édifice mais qui menés avant, ou plutôt à côté de l'étude scientifique, ont eu pour conséquence de figer dans la pierre des interprétations erronées. Nous avons de nombreuses preuves de cela à Mandeure (reprise de maçonnerie, ajout de contrefort sur la façade nord, etc.). A la fin du XIX ${ }^{\mathrm{e}}$ siècle, le théâtre entre véritablement dans le domaine des discussions scientifiques. Les dégagements restent cependant, comparativement à la taille de l'édifice, très partiels et se bornent à une partie de la cavea et de l'orchestra (fig. 3). A cette époque, les façades en grand appareil ne sont pas encore reconnues pas plus que le pulpitum (fig. 4). Lorsque le théâtre de Mandeure sert avec quelques autres à créer le type du théâtre dit gallo-romain, il constitue 
Fig. 4 - Plan du théâtre, synthèse des plans anciens et des relevés topographiques au $1 / 1000$. 
donc un exemplaire d'édifice théâtral sans façade monumentale, ni dispositif scénique! Après une phase assez longue d'abandon, interrompue dans les années cinquante par quelques dégagements plus destructeurs qu'instructifs, il faut attendre les travaux d'Yves Jeannin pour connaître une reprise des recherches sur le théâtre. Il est le premier à restituer une façade avec Theatermotiv sur les premiers niveaux des façades demicirculaires nord et sud. Mais, tributaire de l'état des explorations dans les années soixante et soixante-dix, il n'envisage encore qu'une seule phase de construction qu'il place à l'époque flavienne.

Sur ce point précis de la datation des vestiges architecturaux, il subsiste de nombreuses interrogations. Le phasage des différentes structures est indispensable, faute de quoi on a toutes les chances de mélanger des éléments d'architecture qui n'ont rien à voir entre eux. Malheureusement, le peu de mobilier archéologique découvert ne permet guère de proposer une datation précise des différentes phases de construction et/ou de restauration du théâtre.

Les travaux considérables qui ont été entrepris tant pour la construction des façades à arcades du dernier état que pour les nouveaux aménagements de la cavea ont, en grande partie, recouvert les états antérieurs et, souvent aussi, détruit les stratigraphies plus anciennes. Dans le même ordre d'idées, il faudrait ajouter que les fouilles ou les dégagements pratiqués au XIX $\mathrm{X}^{\mathrm{e}}$ et au $\mathrm{XX}^{\mathrm{e}}$ siècle ont également été assez destructeurs. Il n'en demeure pas moins que des vestiges subsistent çà et là et certains sondages nous ont permis de faire des observations utiles pour reconstituer telle ou telle période de l'histoire de l'édifice.

Par exemple, un sondage ouvert à l'extrémité sud du premier couloir de précinction a montré de manière indubitable que l'édifice de spectacle avait connu au moins trois grandes phases de construction, ayant, pour les deux dernières, entraîné des modifications substantielles du plan. Pour les phases plus anciennes, la présence d'éléments de couronnement en remploi atteste leur caractère monumental, de même d'ailleurs que la présence de blocs de grès, matériau qui a complètement disparu de la phase la plus récente. En outre, d'anciennes tranchées de récupération de matériau de construction, d'époque moderne, ont été vidées au niveau du mur arrière de l'orchestra et sous la basilique sud, permettant ainsi d'observer la reprise du mur sud de la basilique sur toute sa hauteur. Un démontage partiel d'un de ces murs nous a d'ailleurs permis de confirmer leur renforcement substantiel dans la dernière phase 
Fig. 5 - Photo aérienne des fouilles de la première précinction, avec en grisé les murs de l'état antérieur.

d'aménagement du théâtre. Enfin, l'orchestra a connu d'importantes modifications (fig. 5), en plan (une arène de forme ovale aurait précédé l'orchestra circulaire) et en taille (l'orchestra actuellement visible est manifestement atrophiée, surtout si l'on considère l'ampleur exceptionnelle 
de la cavea). Ces transformations s'accompagnent d'un creusement du niveau de l'orchestre afin de pouvoir asseoir une nouvelle rangée de gradins, creusement contemporain de l'exhaussement des niveaux de sol des précinctions sur plus d'1,50 m (fig. 6). L'étude des séries lapidaires attribuées au théâtre confirme ces observations et signale l'existence de deux phases distinctes de décoration en grand appareil.

Pour des raisons documentaires, il sera davantage question dans les lignes qui suivent du dernier état de construction et de décoration du théâtre, les états antérieurs seront abordés succinctement.

\section{Les états antérieurs}

Il est dans l'état actuel de nos recherches rigoureusement impossible de présenter un plan et une élévation de chacune des grandes phases de construction, rien qui puisse être comparable par exemple au plan du théâtre d'Augst qui distingue nettement trois phases ${ }^{4}$. Cette référence à Augst n'est pas fortuite puisqu'il n'est pas impossible qu'à Mandeure aussi l'orchestra actuelle ait pu succéder à une arène. Pour cette phase importante de l'histoire architecturale de l'édifice de spectacle, nous ne disposons que de quelques éléments. Il s'agit tout d'abord des vestiges conservés in situ, d'une orchestra au dessin bien différent de celui qu'elle présente actuellement. Les sondages ouverts entre la première précinction et l'ima cavea ont ainsi permis d'observer, sous le balteus aval de la première précinction, un mur plus ancien, coupé au niveau de la basilique, qui prolonge le demi-cercle en une ellipse (fig. 5). Bien que les constructeurs aient partiellement conservé ces murs pour y appuyer la maçonnerie des murs du dernier état, ils en ont modifié l'orientation. L'inflexion des murs de ce premier théâtre prouve l'existence d'un état dans lequel l'orchestre empruntait un plan ovalaire, sans qu'il soit possible d'en restituer le parcours complet - ouvert ou fermé. Ces murs en opus vittatum soigné présentent une hauteur de parement qui pourrait évoquer des murs de balteus assez hauts. La configuration de la cavea est sans doute déjà assez proche de celle que l'on connaît dans le dernier état, au moins pour ce qui concerne la media cavea. Ainsi, dans la

4. Th. Hufschmid, «La ville romaine d'Augusta-RauricalAugst (Bâle-campagne, $\mathrm{CH})$ et son théâtre». 
Fig. 6 - Axonométrie de l'exhaussement de la précinction grâce à une rampe composée de remplois d'architecture. 
première précinction, l'exhaussement du niveau de circulation s'accompagne de l'exhaussement des accès principaux. Il n'est pas absurde de penser que ce couloir qualifié de couloir de précinction dans son dernier état, tenait lieu dans un premier temps d'ambulacre. Cette précinction fut en effet entièrement comblée et le niveau de circulation considérablement exhaussé grâce à la construction d'une rampe dont la mise en œuvre comprenait de nombreux blocs d'architecture en remploi. Une grande partie de ces fragments de blocs architectoniques et de blocs courants sont en calcaire, mais une quantité non négligeable de fragments de blocs de grès concassés a été découverte. Ceux-ci présentent des caractéristiques techniques, les faces de joint clavées notamment, qui pourraient les rapprocher de blocs de gradins. Si les belles découvertes du théâtre de Dalheim confirment l'existence de sièges de proédrie taillés dans ce matériau, il conviendrait d'explorer aussi cette piste. Parmi les fragments d'architecture, la série des éléments de couronnement à moulures lisses constitue la plus importante en nombre (fig. 7). Les blocs sont tous fragmentaires et ne permettent pas de connaître leur profondeur. L'un d'entre eux présente un angle sortant. Leur lit d'attente, grossièrement travaillé au pic, attesterait leur insertion dans une maçonnerie de petit appareil plutôt que dans une élévation en grand appareil. Leur profil composé d'une série de moulures lisses, où se succèdent talon, listel et doucine, était entièrement stuqué. Ces éléments architecturaux pourraient avoir été disposés en couronnement sur le petit appareil des murs des aditus ou des ambulacres, mais on ne peut exclure un éventuel couronnement de podium. Un autre fragment architectural découvert en un seul exemplaire pourrait, quant à lui, correspondre à la partie sommitale d'un des blocs de parapet ${ }^{5}$. Il faut enfin ajouter à ce catalogue deux fragments de gradins inscrits, le premier qui correspondrait sans doute à un nom propre et l'autre à un numéro d'emplacement, et divers blocs d'emmarchement. Parmi les points remarquables de ce lot, il faut souligner l'homogénéité typologique de ces remplois qui évoque une modification du plan et de l'élévation de l'orchestre et de la partie inférieure de la cavea. Ce premier état reconnu constitue, dans l'état actuel de nos connaissances, le plus ancien du théâtre. Que faut-il en penser? Les vestiges de cette première orchestra suggèrent l'existence d'un mur

5. Son profil sommital en demi-cercle est précédé d'une moulure en talon et bandeau sur un des parements. 
Fig. 7 - Blocs de couronnement en calcaire, découverts en remploi dans la première précinction. 
introduisant une nette dénivellation, plus d'1 $\mathrm{m}$ assurément, entre cavea et orchestra. Ce dispositif se révèle des plus courants dans l'architecture des théâtres des Gaules, à Beaumont-sur-Oise, Ribemont-sur-Ancre, Vieux ou Augst par exemple, mais aussi dans certains théâtres d'Italie ${ }^{6}$. Cette transformation de l'orchestre pour l'adapter à de nouveaux types de jeux serait un phénomène courant à la fin du I $^{\text {er }}$ siècle de notre ère, observé dans les provinces orientales de l'Empire comme dans les provinces occidentales ${ }^{7}$. Cependant à Mandeure, aucun critère chronologique ne permet, pour l'instant, d'asseoir la datation de cette phase d'aménagement du théâtre.

Comme c'est presque toujours le cas, c'est le dernier état qui se laisse le plus aisément appréhender, tant pour le plan que pour les élévations des façades.

\section{Le dernier état}

Dans son dernier état, le théâtre présente une cavea divisée en quatre maeniana et à l'intérieur de laquelle se développe une circulation à la fois ascendante et descendante. Le sommet de la cavea était surmonté d'une porticus in summa cavea, interrompue au centre par une structure qui pourrait être interprétée - ce n'est encore qu'une hypothèse - comme un sacellum. L'orchestra, en forme de demi-cercle outrepassé, est composée de belles dalles de calcaire. Ce plan trouve de nombreux parallèles parmi les théâtres des Gaules (Alésia, Drevant, Jublains, Sanxay, Bouzyla-Forêt, Valognes, Naintré, etc.). D’après les observations de nos prédécesseurs, seul le pulpitum correspondant à une avancée rectangulaire de plus de $9 \mathrm{~m}$ sur $3 \mathrm{~m}$, partiellement maçonnée, est conservé. Il était sans doute destiné à supporter une structure en bois ${ }^{8}$.

Ce dernier état visible résulte d'un processus de transformation du premier édifice qui s'est déroulé en plusieurs étapes. La chronologie relative des structures fouillées dans la partie basse du théâtre introduit

6. F. Dumasy, «Les édifices théâtraux de type gallo-romain, essai de définition ».

7. J.-Ch. Moretti, "L'adaptation des théâtres de Grèce aux spectacles impériaux"; F. Dumasy, "Les édifices de spectacle en Gaule du Nord".

8. Une partie du dispositif scénique a été fouillée en août 2010 et fera l'objet de publications ultérieures. 


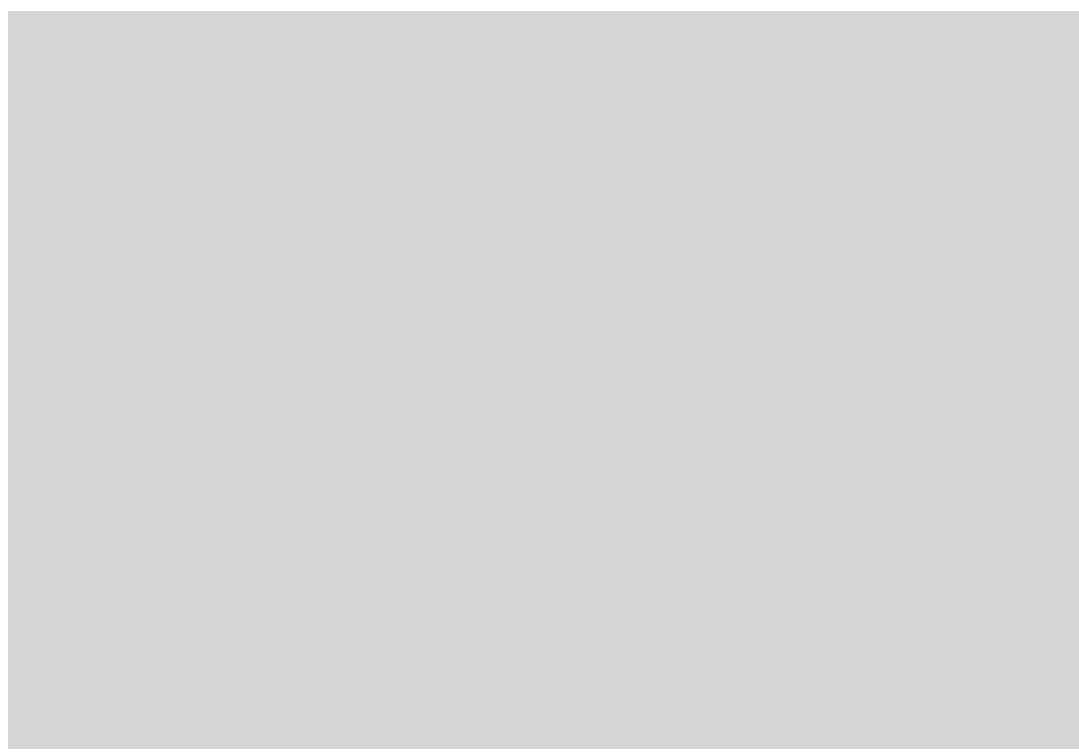

Fig. 8 - Comblement de la dernière arcade de la façade diamétrale, état avant restauration.

plusieurs observations intéressantes sur ce point. Au moins une étape intermédiaire impose un plan proche de celui dont nous disposons actuellement, c'est-à-dire l'implantation d'une orchestra hémicirculaire, et de basiliques de part et d'autre d'un pulpitum réduit, ainsi que la construction d'un mur sur toute la longueur de la façade diamétrale. Les élévations ne présentent sans doute pas la même ambition que dans le dernier état. Les arcades du mur diamétral sont déjà en place, selon une séquence de dix arcades côté nord et dix côté sud. L'élévation des murs des façades hémicirculaires de cette phase est inconnue. La dernière phase de construction reconnue correspond à l'achèvement du programme établi précédemment, qui se concentre cette fois sur la mise en œuvre des façades en grand appareil. Le programme de planification générale ne change guère, mais l'effort porte sur un renforcement systématique des substructions comme des élévations. Ainsi la maçonnerie des murs de la façade diamétrale est doublée comme celle des aditus nord et sud, ainsi que celle des murs des basiliques. Les deux arcades de la façade diamétrale qui flanquent le mur de scène sont comblées (fig. 8), réduisant ainsi le rythme d'arcades de dix à neuf de part et d'autre. Selon les observations réalisées récemment sur le mur arrière du théâtre, 
il subirait lui aussi des modifications importantes afin de supporter ces grandes façades.

La dernière phase de construction, assimilable à la dernière tranche des travaux initiés plus tôt, rend compte de l'ambition architecturale considérable placée dans cet édifice. Après la mise en ouvre d'une façade diamétrale de $142 \mathrm{~m}$ de long, dimension qui place le théâtre de Mandeure parmi les deux plus grands théâtres de Gaules, l'ambition édilitaire se porte sur les façades décorées d'un Theatermotiv qui se développe sur deux, voire trois niveaux. Il reste malheureusement impossible de dater précisément ces deux dernières phases de construction. Pour ce dernier état, qui est naturellement le mieux connu, la restitution des façades a connu des progrès décisifs depuis la première hypothèse de restitution réalisée par Yves Jeannin ${ }^{9}$. L'étude architecturale et l'attribution de nombreux fragments de blocs erratiques ont permis de confirmer la restitution d'un système d'arcades pour le premier niveau des deux façades hémicirculaires et pour la façade diamétrale mais également, sur au moins deux niveaux supplémentaires, pour les façades semicirculaires nord et sud. Les étapes de la restitution des façades, fondées sur les vestiges conservés en place et les fragments d'architecture, ont déjà été présentées à l'occasion d'autres publications, c'est pourquoi elles ne seront ici que brièvement exposées ${ }^{10}$. Les vestiges d'un stylobate en grand appareil conservé en place sur les façades hémicirculaires nord et sud attestent la présence de piédroits de colonnes engagées disposées régulièrement tous les 4,14 $\mathrm{m}$ le long de la façade sur le premier niveau. $\mathrm{Au}$ deuxième niveau, les vestiges conservés confirment un dispositif en tout point similaire (fig. 9). L'entraxe des colonnes et les dimensions du soubassement en grand appareil sont identiques. Sur la façade diamétrale en revanche, la configuration est très légèrement différente puisque le rythme des arcades présente un entraxe un peu plus grand: il est de $4,32 \mathrm{~m}$. A l'observation des vestiges conservés in situ, il faut ajouter un certain nombre de blocs épars, venant de découvertes anciennes ou récentes, dont plusieurs séries ont pu être rapprochées du théâtre. L'état de conservation de ces blocs est très variable. Il s'agit pour la majorité d'entre eux d'éléments de décors bûchés pour être remployés.

9. Y. Jeannin, «Le théâtre de Mandeure».

Io. J.-Y. Marc et al., «Le complexe du sanctuaire et du théâtre de Mandeure (Doubs, F), de ses origines gauloises à sa monumentalisation romaine». 
LE THÉÂTRE DE MANDEURE

63

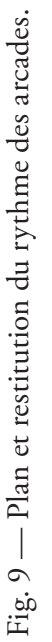


Fig. 10 - Hypothèse de restitution des arcades.

Parmi les séries qui appartiennent à ce dernier état monumental (fig. 10), on dénombre une quantité importante de blocs appartenant aux éléments verticaux (base, fût de colonne et chapiteau). Les bases engagées présentent un profil attique classique. Elles ne reposent pas sur une plinthe. Un léger scamillus, qui peut mesurer de 1 à $1,50 \mathrm{~cm}$ de haut, est aménagé au niveau de leur lit de pose. Plusieurs éléments de fûts de colonnes engagées d'un diamètre variant de 0,55 à 0,59 m assurent la restitution d'un fût rudenté. Un des blocs-clés de la restitution présente à la fois une colonne engagée flanquée de part et d'autre par les départs d'archivolte (fig. 11). Enfin, des éléments de chapiteaux, on ne conserve que deux éléments de chapiteaux probablement corinthiens ou 


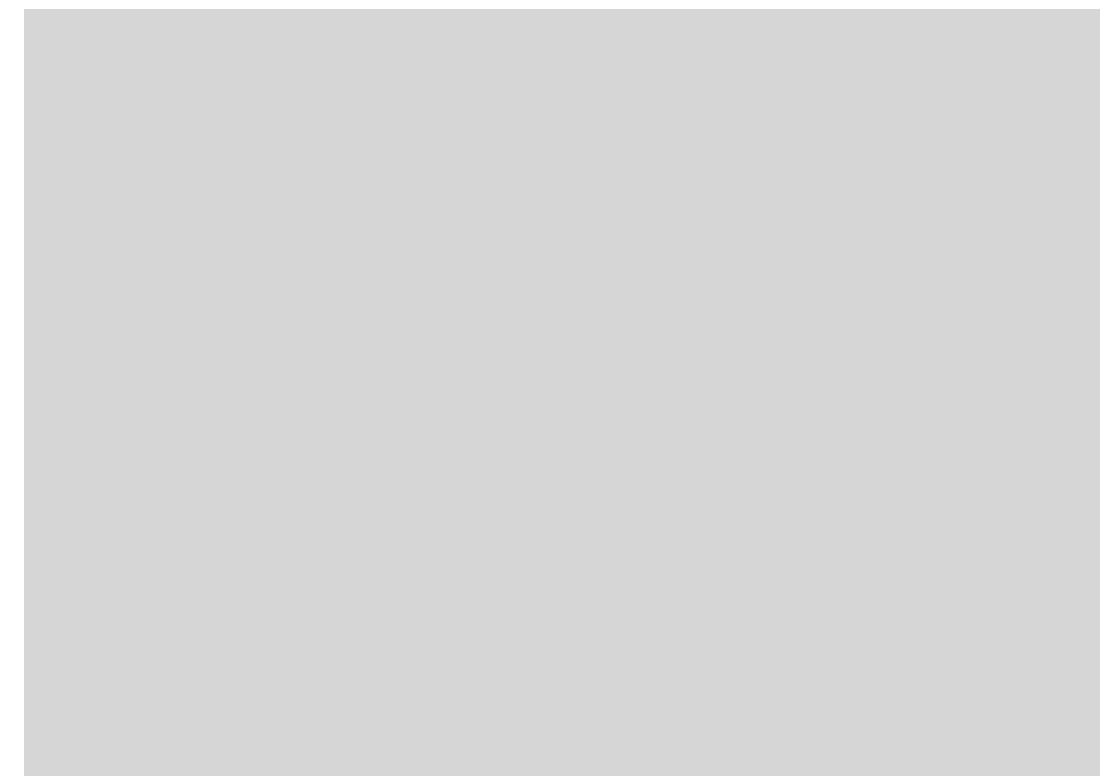

Fig. 11 — Fût de colonne rudentée avec départ d'archivolte au moment de sa découverte.

composites laissés à l'état d'épannelage, mais dont les mortaises de bardage et de scellement témoignent de leur mise en place. Les claveaux qui sont conservés attestent que les arcades mettaient en œuvre deux cours de claveaux. La première à parement lisse tournée vers l'intérieur des couloirs et la deuxième, à parement orné de bandeaux lisses surmontés d'un couronnement en talon et bandeau, tournée vers l'extérieur. Des éléments horizontaux, qui appartiennent à cette séquence décorative, permettent de restituer l'entablement dans son intégralité. Leurs moulures reprennent les cartons classiques de l'architecture des édifices de spectacle. L'architrave à trois faces séparées par des moulures de transition lisses est surmontée d'un couronnement en talon et bandeau. La frise à parement lisse est composée de blocs de clé et de contre-clé destinés à soulager l'architrave. Enfin, la corniche modillonnaire reprend un type à moulures lisses et à caissons ornés de prismes tronqués, bien connu des élévations des édifices de spectacle à Nîmes, Arles ou Alésia ${ }^{11}$.

II. A. Olivier, «Corniches et couronnements gallo-romains à Alésia (Alise-SainteReine, Côte d'Or)». 
66

ÉTUDES DE LETTRES

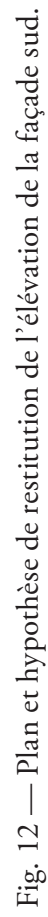




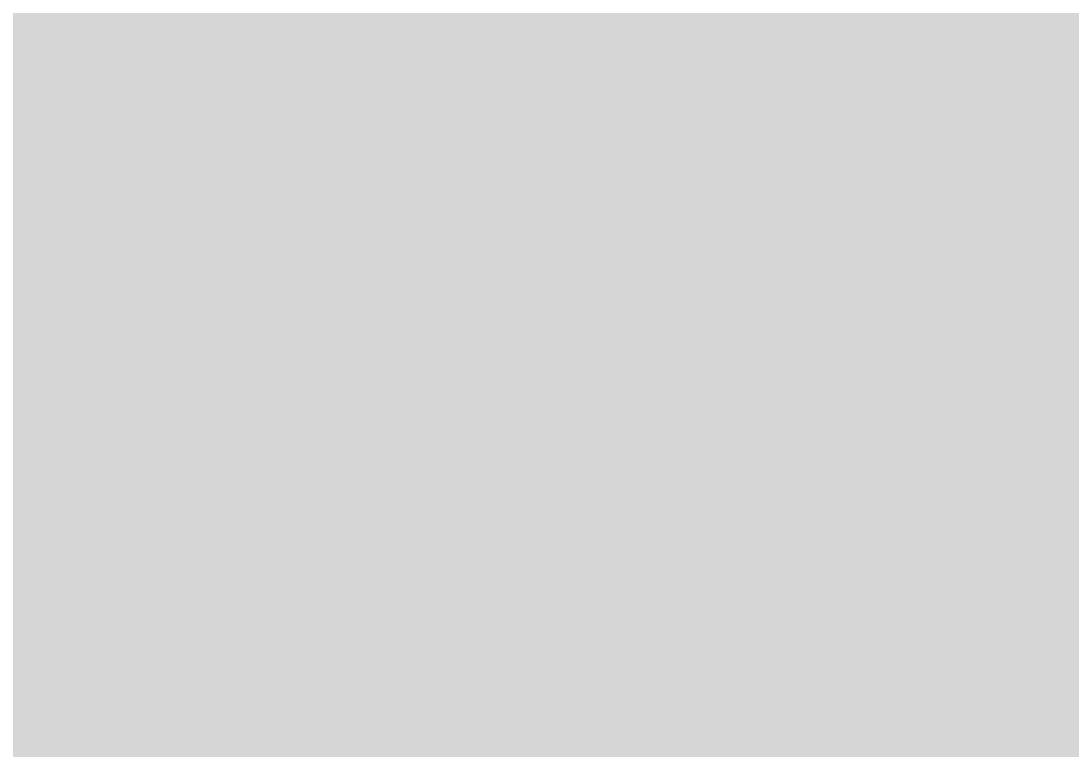

Fig. 13 - Base et fût de colonne découverts lors des dégagements à l'arrière du pulpitum.

Si la restitution des deux premiers niveaux d'arcades est validée tant par les vestiges en place que par les fragments du décor, de grandes difficultés, liées autant à l'état de la documentation qu'à nos recherches, persistent. Pour les façades semi-circulaires, la hauteur conservée de la cavea ainsi que le mur arrière du théâtre scandé de pilastres en ressaut dans la maçonnerie en petit appareil incitent à restituer un, voire deux niveaux supplémentaires d'élévation (fig. 12). Le dispositif précis reste cependant difficile à restituer. Des fragments de frises-architravées, reprenant l'ornementation des deux premiers niveaux, mais dans un module plus petit, ont été découverts au pied des façades nord et sud du théâtre. Ils permettent de proposer la restitution d'un étage supplémentaire, composé d'un ordre de pilastres surmontés d'un entablement à ressaut, qui pourrait convenir à un troisième niveau. La fouille de la zone située derrière le mur arrière du théâtre permettra sans doute d'apporter des informations précieuses concernant les niveaux de sol et l'éventuel niveau de départ de cet ordre à pilastres. La façade diamétrale constitue l'autre point délicat de la restitution. Le dossier comparatif n'est sur ce point d'aucune aide. Les restitutions divergent largement d'un monument à l'autre et constituent l'illustration la plus éloquente 
des diverses interprétations dont fait l'objet le théâtre gallo-romain. Pour certains, il s'agit d'ouvrir cette façade diamétrale pour que les spectateurs bénéficient d'une vue sur le temple situé en face et pour d'autres, il faut restituer un mur de scène plus ou moins modeste selon les cas. A Mandeure, des arcades ornent la façade jusqu'à la limite des premières précinctions où elles laissent la place à un mur construit en petit appareil de $0,80 \mathrm{~m}$ d'épaisseur et solidement fondé à $2 \mathrm{~m}$ de profondeur. Comment interpréter ce dispositif? Le théâtre, considéré a priori comme relevant plutôt d'une fonction religieuse, n'avait pas vraiment été étudié comme un édifice de spectacle. On a alors admis que l'équipement scénique du théâtre était réduit à sa plus simple expression. Or, ce mur qui pouvait aisément s'élever à une hauteur de 4 ou $5 \mathrm{~m}$ signale une fermeture effective de l'orchestra, à l'arrière du pulpitum. Une fois encore, ce mur et son articulation avec les arcades ne sont pas aisés à restituer en élévation. Les seuls éléments qui pourraient évoquer la décoration de cette partie du théâtre ont été découverts au cours des dégagements particulièrement destructeurs du milieu du XXe siècle. Il s'agit de fragments d'une colonne libre en calcaire blanc (base de colonne et fût lisse) d'un module très modeste puisque le diamètre en est de $0,29 \mathrm{~m}$ (fig. 13).

\section{Conclusion}

Ces premières considérations illustrent combien le concept de «théâtre gallo-romain» et les cadres qu'il impose sur le plan typologique et fonctionnel perturbent le processus d'interprétation. Comme on l'a vu, le théâtre de Mandeure présente toutes les caractéristiques de la monumentalité la plus imposante, celle en tous cas qui est la marque des théâtres ou des amphithéâtres dans le monde romain: façades à arcades, Theatermotiv, chapiteaux corinthiens et corniches modillonaires, etc. Il est vrai que le dispositif scénique, le pulpitum et l'orchestra en particulier, apparaît ici sous une forme atténuée. Mais tout semble indiquer que l'orchestra n'était pas ouverte sur le sanctuaire et qu'elle était, au contraire, limitée par un dispositif architectural continu. En revanche, s'il n'y a plus, à nos yeux du moins, de liens visuels entre les spectateurs assis au théâtre et le sanctuaire, situé à $200 \mathrm{~m}$ de distance, il n'en est pas moins vrai que ces deux édifices entretiennent une sorte de dialogue monumental et que cette relation doit être désormais comprise en prenant en 
compte un complexe sacré beaucoup plus riche et beaucoup plus vaste. Ce binôme ne constitue qu'une partie d'un complexe monumental qui s'étend sur plus de 15 ha. Nous voudrions terminer en insistant sur le fait que ce qui vient de vous être présenté doit être considéré encore comme une suite d'hypothèses et que, tant que l'étude monographique et exhaustive de cette construction ne sera pas menée à bien, il serait bien imprudent d'en retirer des certitudes.

Séverine BLIN

Institut de Recherches sur l'Architecture Antique, Université de Lyon II

Jean-Yves MarC

Institut d'Archéologie Classique, Université de Strasbourg - UMR 7044 


\section{BIBLIOGRAPHIE}

BLIN, Séverine, "Architecture et société à Mandeure au Haut-Empire», Bulletin de la Société d'Emulation de Montbéliard, 131 (2008), p. 23-48.

Dumasy, Françoise, «Les édifices théâtraux de type gallo-romain, essai de définition", Latomus, XXXIV, 4 (1975), p. 1010-1019.

—, "Les édifices de spectacle en Gaule du Nord. De la typologie à la chronologie", in Les villes romaines du Nord de la Gaule. Vingt ans de recherches nouvelles, actes du XXV $V^{e}$ colloque international de Halma-Ipel, éd. Roger Hanoune, Villeneuve-d'Ascq, Revue du Nord, 2007, p. 447-466.

FrÉzouls, Edmond, «Problèmes archéologiques du théâtre romain», in Atti del III congresso di studi sul dramma antico, Siracusa, 22-24 maggio 1969, Roma/Siracusa, Istituto nazionale del dramma antico, 1971, p. 139-156.

— «Larchitecture du théâtre romain en Italie», Bolletino del Centro di Storia dell'Architettura Andrea Palladio, 16 (1974), p. 35-71.

— "Aspects de l'histoire architecturale du théâtre romain", Aufstieg und Niedergang der römischen Welt, 2.12 .1 (1982), p. 425-435.

Hufschmid, Thomas, "La ville romaine d'Augusta-RauricalAugst (Bâle-campagne, $\mathrm{CH}$ ) et son théâtre. Les recherches actuelles", in Premières journées archéologiques frontalières de l'Arc jurassien. Mandeure, sa campagne et ses relations d'Avenches à Luxeuil et d'Augst à Besançon, dir. Cécile Belet-Gonda, Presses Universitaires de Franche-Comté et Porrentruy, 2007, p. 59-72.

Jeannin, Yves, «Le théâtre de Mandeure: le problème de sa décoration architecturale», Archéologia, 62 (1973), p. 39-48.

Marc, Jean-Yves, André, Nathalie, Barral, Philippe, Blin, Séverine, Bossuet, Gilles, Joly, Martine, Mougin, Pierre, Thinet, Matthieu, «Le complexe du sanctuaire et du théâtre de Mandeure (Doubs, F), de ses origines gauloises à sa monumentalisation 
romaine", in Premières journées archéologiques frontalières de l'Arc jurassien. Mandeure, sa campagne et ses relations d'Avenches à Luxeuil et d'Augst à Besançon, dir. Cécile Belet-Gonda, Presses Universitaires de Franche-Comté et Porrentruy, 2007, p. 13-34.

Matter, Michel, «Particularités architecturales des édifices de spectacle en Gaule lyonnaise", in Spectacula II. Le théâtre antique et ses spectacles. Actes du colloque tenu au musée archéologique Henri-Prades de Lattes les 27, 28, 29 et 30 avril 1989, éd. Christian Landes, Lattes, Imago, 1992, p. 29-36.

Moretri, Jean-Charles, "L'adaptation des théâtres de Grèce aux spectacles impériaux", in Spectacula II. Le théâtre antique et ses spectacles. Actes du colloque tenu au musée archéologique Henri-Prades de Lattes les 27, 28, 29 et 30 avril 1989, éd. Christian Landes, Lattes, Imago, 1992, p. 179-186.

Olivier, Albéric, "Corniches et couronnements gallo-romains à Alésia (Alise-Sainte-Reine, Côte d'Or)», Gallia, 46 (1989), p. 46-69.

TosI, Giovanna, Gli edifici per spettacoli nell'Italia romana, Roma, Quasar, 2003.

\section{Crédits iconographiques}

Fig. 1:

PCR Mandeure 2008.

Fig. 2:

PCR Mandeure 2008. Montage et DAO S. Blin, d'après les plans de G. Bossuet, M. Thivet, J. Monnier, PCR.

Fig. 3:

D’après F. Morel-Macler, Les Antiquités de Mandeure, Montbéliard, 1847.

Fig. 4:

PCR Mandeure 2009.

Fig. 5:

Séverine Blin. 
Fig. 6, 9 et 12:

Nathalie André.

Fig. 7:

Jean-Yves Marc.

Fig. 8 et 11 :

Yves Jeannin.

Fig. 10:

Eleonore Cren.

Fig. 13:

Lucien Lerat. 